\title{
ON THE EXTREME POINTS OF CLASSES OF UNIVALENT FUNCTIONS
}

\author{
HEIKKI HAARIO
}

\section{Introduction}

Let $H(D)$ denote the set of all analytic functions in the unit disc $D=\{z \in C \mid$ $|z|<1\}$. Equipped with the usual topology of locally uniform convergence $H(D)$ is a locally convex topological vector space. A function $f \in B$ is called an extreme point of a subset $B \subset H(D)$ if it cannot be written as a proper convex combination of two distinct elements of $B$. According to the Krein-Millman theorem the extreme points of a compact set $B$ span the closed convex hull $\overline{\mathrm{co}}(B)$ of $B$, and in the case of a compact $\overline{\mathrm{co}}(B)$ the extreme points of $\overline{\mathrm{co}}(B)$ belong to $B$. Each continuous linear functional on $H(D)$ achieves its extremums in a compact set $B$ at some extreme point of $\overline{c o}(B)$. We call $f \in B$ a support point of the set $B$ if there is a nonconstant continuous linear functional which achieves its maximum at $f$.

Denote by $S$ the compact set of $H(D)$ consisting of all univalent functions in $D$, normalized by $f(0)=0, f^{\prime}(0)=1$. L. Brickman showed that every extreme point of $S$ maps $D$ onto the exterior of a slit with monotonically increasing modulus [3], but a complete characterization of the extreme points of $S$ is not known. On the other hand, the extreme points of most of the common subclasses of $S$ (starlike, convex, close-to-convex functions, functions convex in one direction, functions with real coefficients) have been determined [4], [9]. In each case the extreme points turn out to form a simple set of familiar functions. The extreme point method thus gives an effective tool for linear extremum problems in such classes.

The functions with bounded boundary rotation provide an example of a wellknown subclass of $S$ whose extreme points are still unknown. Let $B_{k}, k \geqq 2$, denote the class of functions

$$
f(z)=z+a_{2} z^{2}+a_{3} z^{3}+\ldots
$$

which are analytic in $D$ and map $D$ onto a domain with bounded boundary rotation. The functions of $B_{k}$ are obtained from the equation

$$
1+z \frac{f^{\prime \prime}(z)}{f^{\prime}(z)}=\frac{1}{2} \int_{0}^{2 \pi} \frac{z+e^{i \varphi}}{z-e^{i \varphi}} d \mu(\varphi),
$$


where $\mu$ ranges over the compact (in the weak $*$ topology) set of real Borel measures on $\partial D$ with

$$
\int_{0}^{2 \pi} d \mu=2, \quad \int_{0}^{2 \pi}|d \mu| \leqq k
$$

By (2) the class $B_{k}$ is a continuous image of a compact set and thus compact. For $k \leqq 4$ the functions in $B_{k}$ are univalent, for $k=2$ the class $B_{k}$ coincides with the class of convex functions.

The convex hull and extreme points of the class $B_{R}(k)$ consisting of $B_{k}$-functions with real coefficients are not known. Here we give a set of extreme points and support points of $B_{R}(k)$ by determining the extreme points of the coefficient body $V_{3}=\left(a_{2}, a_{3}\right)$. In the other subclasses of $S$ listed above the analogous families of functions give precisely the extreme points of the corresponding classes with real coefficients. However, by studying linear functionals on the body $V_{4}$ we see that in the classes $B_{R}(k)$ there are more extreme points than those given by $V_{3}$. This indicates that, in contrast to the situation in the other well-known subclasses of $S$, the extreme points of $B_{R}(k)$ are too numerous to be of value.

Integration of (2) with discrete measures gives the Schwarz-Christoffel mappings. Especially, integration with a measure supported by two points $x, y$ $x \neq y,|x|=|y|=1$, yields the functions

$$
f_{k}(z, x, y)=\frac{2}{k(x-y)}\left(\left(\frac{1+x z}{1+y z}\right)^{k / 2}-1\right) .
$$

In [10] Hengartner, Pfluger and Schober showed that for $k \geqq 4$ the support points of $B_{k}$ are of the form (3) and conjectured that the same holds for $2<k<4$. In favour of this conjecture they showed that linear functionals on the body $V_{3}$ of $B_{k}$ are maximized only by the functions (3). Here we study more closely which of the functions (3) indeed can give a maximum for a linear functional of $a_{2}, a_{3}$. It turns out that only functions which are close enough to the "Koebe functions" of the class $B_{k}$, i.e., the functions (3) with $x=-y$, can do this.

It is known that in the class $S$ each support point maps the unit disc onto the complement of a single analytic arc with the so called $\pi / 4$ property [11]. Examples of support points of $S$ can be obtained by a study of linear functionals of $a_{2}, a_{3}$. Using the Lowner method we get, similarly to the case of $B_{k}$, a certain "neighbourhood" of the Koebe function in which such functionals only can reach their maxima. 


\section{On the extreme points of the class $B_{R}(k)$}

By aid of the Herglotz integral representation formula the convex hulls and extreme points of several subclasses of $S$ were determined in [4], [9]. We recall the results for some of these classes. Denote by $S_{R}$ the class of univalent functions with real coefficients and by $S t_{R}, K_{R}, C_{R}, F_{R}$ the classes of functions with real coefficients which are starlike, convex, close-to-convex or convex in the direction of the imaginary axis. Let $X=\{z \in C,|| z \mid=1, \operatorname{Im} z \geqq 0\}$, and let $P$ denote the set of probability measures on $X$. If $T$ denotes the set of (typically real) functions

$$
f_{\mu}(z)=\int_{X} \frac{z}{(1-x z)(1-\bar{x} z)} d \mu(x), \quad \mu \in P,
$$

then $\overline{\mathrm{co}}\left(S_{R}\right)=\overline{\mathrm{co}}\left(S t_{R}\right)=\overline{\mathrm{co}}\left(C_{R}\right)=T$, and the extreme points of these classes are precisely the functions $z /(1-x z)(1-\bar{x} z), x \in X$.

Similarly, the closed convex hull of $K_{R}$ and $F_{R}$ is the set of functions

$$
f_{\mu}(z)=\int_{X} \frac{1}{x-\bar{x}} \log \frac{1-x z}{1-\bar{x} z} d \mu(x), \quad \mu \in P,
$$

and the extreme points of $\overline{\mathrm{co}}\left(K_{R}\right)$ and $\overline{\mathrm{co}}\left(F_{R}\right)$ are precisely the functions $(x-\bar{x})^{-1} \log ((1-x z)(1-\bar{x} z)), x \in X$; see [9]. Below we give a set of extreme points in the class $B_{R}(k)$, from which the extreme points of $\overline{c o}\left(K_{R}\right)$ are obtained as a special case with $k=2$.

It is evident that in any class of functions for every extreme point $\left(a_{2}, \ldots, a_{n}\right)$ of the body $V_{n}, n \geqq 2$, the corresponding boundary function of the body is an extreme point of the class: otherwise the representation $f(z)=t f_{1}(z)+(1-t) f_{2}(z)$ would imply that the point $\left(a_{2}, \ldots, a_{n}\right)$ would not be an extreme point of $V_{n}$. In this way we can construct examples of extreme points in any class of functions by identifying the extreme points of coefficient bodies. Since in $S$ and its subclasses each $V_{n}$ has a non-empty interior, every boundary point of $\overline{c o}\left(V_{n}\right)$ has a non-constant supporting functional. Because $E\left(\overline{c o}\left(V_{n}\right)\right) \subset V_{n}$, the extreme points of the class corresponding to the extreme points of $\overline{\mathrm{co}}\left(V_{n}\right)$ are thus support points of the class, too (generally it is only known that the extreme points of the closed convex hull belong to the closure of the set of support points [5]). It can be expected that in classes where the extreme points are relatively few a considerable amount of them is produced by the initial bodies already. The first (trivial) region $V_{2}$ of starlike and convex functions is given by $\left|a_{2}\right| \leqq 2$ and $\left|a_{2}\right| \leqq 1$, respectively. The functions corresponding to the boundary points, the Koebe mapping with its rotations and the functions $z /(1-x z),|x|=1$, are thus extreme points of the classes of starlike and convex functions. In these classes the boundary functions of $V_{2}$ actually exhaust the set of extreme points cf. [4]. In classes with real coefficients the determination of the extreme points of $V_{3}$ is equally straightforward. 
Theorem 1. Let $B_{R}(k)$ be the class of $B_{k}$-functions with real coefficients. For any $k \geqq 2$ the functions

$$
\begin{aligned}
& f(z)=\int_{0}^{z} \frac{(1+t)^{v}(1-t)^{k / 2-1-v}}{\left(1+t^{2}\right)^{k / 4+1 / 2}} d t, \quad 0 \leqq v \leqq k / 2-1, \\
& f(z)=\int_{0}^{z} \frac{(1+t)^{k / 2-1}}{[(1-x t)(1-\bar{x} t)]^{k / 4+1 / 2}} d t, \quad \arg x \in[0, \pi / 2], \\
& f(z)=\int_{0}^{z} \frac{(1-t)^{k / 2-1}}{[(1-x t)(1-\bar{x} t)]^{k / 4+1 / 2}} d t, \quad \arg x \in[\pi / 2, \pi]
\end{aligned}
$$

are extreme points of $\overline{\mathrm{co}}\left(B_{R}(k)\right)$ and support points of $B_{R}(k)$.

Proof. The upper and lower boundaries of $a_{3}$ in terms of $a_{2}$ read

$$
\begin{aligned}
& a_{3} \leqq \frac{2}{3} a_{2}^{2}+\frac{k}{6}, \text { for } 2\left|a_{2}\right| \leqq k / 2+1, \\
& a_{3} \leqq \frac{2}{3} \frac{k-6}{k-2} a_{2}^{2}+\frac{4}{3} \frac{k+2}{k-2}\left|a_{2}\right|-\frac{3 k+2}{3(k-2)}, \text { for } k / 2+1 \leqq 2\left|a_{2}\right| \leqq k, \\
& a_{3} \geqq \frac{2}{3} a_{2}^{2}-\frac{k}{6}, \text { for } 2\left|a_{2}\right| \leqq k / 2-1, \\
& a_{3} \geqq \frac{2}{3} \frac{k+6}{k+2} a_{2}^{2}-\frac{4}{3} \frac{k-2}{k+2}\left|a_{2}\right|+\frac{-3 k+2}{3(k+2)}, \text { for } k / 2-1 \leqq 2\left|a_{2}\right| \leqq k ;
\end{aligned}
$$

cf. [7] where the complex body $V_{3}$ of $B_{k}$ was determined. By the formulas (5) the extreme points of $\overline{\mathrm{co}}\left(V_{3}\right)$ for any $k \geqq 2$ are just the points on the lower boundary of $V_{3}$, and so the corresponding functions are extreme points of $\overline{\mathrm{co}}\left(B_{R}(k)\right)$ and support points of $B_{R}(k)$.

The expressions (4) we get from (2) by recalling that for $2\left|a_{2}\right| \leqq k / 2-1$ equality on the lower boundary occurs with a measure $\mu$ with two equal positive jumps of size $k / 4+1 / 2$ at the points $\varphi=\pi / 2$ and $\varphi=3 \pi / 2$, and two negative jumps $-v,-k / 2+1+v, 0 \leqq v \leqq k / 2-1$, at the points $\varphi=0$ and $\varphi=\pi$. For $2\left|a_{2}\right| \geqq k / 2-1$ the support of the measure for the lower boundary consists of three points: there is a negative jump of size $-k / 2+1$ at $\varphi=0$ or $\varphi=\pi$, and two equal positive jumps $k / 4+1 / 2$ at $\varphi= \pm \varphi_{1}$, where $\varphi_{1} \in[\pi / 2, \pi]$ or $\varphi_{1} \in[0, \pi / 2]$, respectively.

Remark 1. For $k=2$ the class $B_{R}(k)$ coincides with the class $K_{R}$. For $k=2$ the first type of the functions (4) shrinks away, and the two last types yield exactly the functions $(x-\bar{x})^{-1} \log ((1-x z) /(1-\bar{x} z))$, arg $x \in[0, \pi]$. The lower boundary and extreme points of $V_{3}$ in the classes $S_{R}, S t_{R}, K_{R}, C_{R}, F_{R}$ are given by the functions $z /(1-x z)(1-\bar{x} z)$, arg $x \in[0, \pi]$ (which are connected with those of $K_{R}$ by the transformation $\left.f(z) \rightarrow z f^{\prime}(z)\right)$. Thus the extreme points in all the classes $\overline{\mathrm{co}}\left(S_{R}\right), \overline{\mathrm{co}}\left(S t_{R}\right), \overline{\mathrm{co}}\left(K_{R}\right), \overline{\mathrm{co}}\left(C_{R}\right), \overline{\mathrm{co}}\left(F_{R}\right)$ are precisely the functions belonging to the extreme points of $V_{3}$. By the next remark the same is not true for $B_{R}(k)$, so the set of extreme points of $\overline{\mathrm{co}}\left(B_{R}(k)\right)$ is more complex. 
Remark 2. We show that the functions (4) do not exhaust the extreme points of $\overline{\mathrm{co}}\left(B_{R}(k)\right)$ and support points of $B_{R}(k)$ by maximizing functionals of the form $a_{4}+t a_{3}+u a_{2}, t, u \in R$, on the boundary of the region $V_{4}$. The upper boundary $a_{4}=\max a_{4}\left(a_{2}, a_{3}\right)$ consists of nine different parts corresponding to the various types of the generating measure $\mu$; see [8] where the body was determined (the lower boundary is obtained by the transformation $f(z) \rightarrow-f(-z),\left(a_{2}, a_{3}, a_{4}\right) \rightarrow$ $\left.\left(-a_{2}, a_{3},-a_{4}\right)\right)$. Because of the complexity of the formulas giving these parts the search for the maxima had to be done by aid of a computer. A standard program for finding minima (E04JAF from the NAG library) was applied to the functional for different values of $t$ and $u$. In this way several functionals were found which are not maximized among the functions (4). Having found such an example we can readily check the situation directly.

As a typical example, consider the functional $a_{4}-a_{3}$. Its values on the lower boundary of $V_{3}$ are most easily computed if we substitute the measure $\mu$ into the formulas

$$
\begin{aligned}
& 2 a_{2}=\int_{0}^{2 \pi} e^{-i \varphi} d \mu(\varphi), \\
& 6 a_{3}=4 a_{2}^{2}+\int_{0}^{2 \pi} e^{-2 i \varphi} d \mu(\varphi), \\
& 12 a_{4}=-8 a_{2}^{3}+18 a_{2} a_{3}+\int_{0}^{2 \pi} e^{-3 i \varphi} d \mu(\varphi),
\end{aligned}
$$

which follow from the basic equation (2). We get the following cases. For $2 a_{2} \geqq$ $k / 2-1$

$$
\begin{aligned}
2 a_{2} & =k / 2-1+\tau(k / 2+1), \\
6 a_{3} & =4 a_{2}^{2}-k+(k+2) \tau^{2}, \\
12 a_{4} & =-8 a_{2}^{3}+18 a_{2} a_{3}-6 a_{2}+4(k / 2-1)+4 \tau^{3}(k / 2+1),
\end{aligned}
$$

where the parameter $\tau=\cos \varphi, 0 \leqq \tau \leqq 1$, represents the sites of the positive jumps of the measure $\mu$. For $2\left|a_{2}\right| \leqq k / 2-1$

$$
\begin{aligned}
2 a_{2} & =-2 v+k / 2-1, \\
6 a_{3} & =4 a_{2}^{2}-k, \\
12 a_{4} & =-8 a_{2}^{3}+18 a_{2} a_{3}+2 a_{2},
\end{aligned}
$$

where the parameter $v, 0 \leqq v \leqq k / 2-1$, gives the size of the negative jump of $\mu$. The third case is obtained from the first one by a change of signs of $a_{2}$ and $a_{4}$.

Take now, for instance, $k=4$. It is not difficult to check that the maximum occurs in the first case with $\tau=1$. Thus we have $\max \left(a_{4}-a_{3}\right)=1$ among the functions (4).

The global maximum of $a_{4}-a_{3}$ occurs in $B_{R}(4)$ in a part called Case $4 \mathrm{~b}$ in [8]. In this part of the surface $a_{4}=\max a_{4}\left(a_{2}, a_{3}\right)$ the support of $\mu$ consists 
of four points: $\mu$ has negative jumps $-v,-k / 2+1+v, 0 \leqq v \leqq k / 2-1$, at $\varphi=0$ and $\varphi=\pi$, and positive jumps $k / 4+1 / 2$ at the points where $\cos \varphi=\tau,-1 / 3 \leqq \tau \leqq 0$. From (6) one readily computes the expressions

$$
\begin{aligned}
& 2 a_{2}=-2 v+k / 2-1+\tau(k / 2+1), \\
& 6 a_{3}=4 a_{2}^{2}-k+\tau^{2}(k+2), \\
& 6 a_{4}=-4 a_{2}^{3}+9 a_{2} a_{3}+a_{2}-\left(\tau-\tau^{3}\right)(k+2) .
\end{aligned}
$$

The maximum for $k=4$ is achieved with $\tau=-0.2492011 \ldots, v=0.5479380 \ldots$, for which $a_{4}-a_{3}=1.006874 \ldots$.

Similar examples of linear functionals not maximized by the functions (4) were found for all tested $2<k<4$ and $k>4$.

Remark 3. It should be noted that the situation in the classes $B_{R}(k)$ does not necessarily indicate an analogous "splitting" of the extreme points in the classes $B_{k}$. Indeed, for $k=4$ the extreme points of $\overline{c o}\left(B_{k}\right)$ are exactly the functions (3), and for $k>4$ the extreme points of $\overline{\mathrm{co}}\left(B_{k}\right)$ and support points of $B_{k}$ are known to be among the functions (3); see [1], [2], [4], [10].

\section{Linear functionals on the body $V_{3}$ in the classes $B_{k}$ and $S$}

The region $V_{2}$ of $B_{k}$ is given by $\left|a_{2}\right| \leqq k / 2$. The extremal functions are the "Koebe functions" of $B_{k}$, the functions (3) with $x=-y$. We next give a necessary condition for the extension which the body $V_{3}$ can provide to this set of support points of $B_{k}$.

Theorem 2. For $k \geqq 2$ the functional $\operatorname{Re}\left\{\tau^{2} a_{3}-\tau \lambda a_{2}\right\}, \tau, \lambda \in C, \tau=1$, can reach its maximum in $B_{k}$ only with functions of the form (3) satisfying the additional condition

$$
|\arg (-x \bar{y})| \equiv c(k),
$$

where $c(k)$ is a decreasing function of $k$.

Proof. Linear functionals of $a_{2}, a_{3}$ reach their maxima only by measures $\mu$ whose support consists of two points [10]. We fix the notation that the point $\varphi_{1}$ corresponds to the positive jump $\Delta_{1}=k / 2+1$, and the point $\varphi_{2}$ to the negative $\Delta_{2}=-k / 2+1$. Consider for a fixed $k$ the functional $L\left(\varphi_{1}, \varphi_{2}\right)=\operatorname{Re}\left\{6 a_{3}-2 \lambda a_{2}\right\}$ with $\lambda=\lambda_{1}+i \lambda_{2}, \lambda_{1}, \lambda_{2} \in R$. By (6)

(7) $L\left(\varphi_{1}, \varphi_{2}\right)=\left(\sum_{i=1}^{2} \cos \varphi_{i} \Delta_{i}\right)^{2}-\left(\sum_{i=1}^{2} \sin \varphi_{i} \Delta_{i}\right)^{2}+\sum_{i=1}^{2}\left(\cos 2 \varphi_{i}-\lambda_{1} \cos \varphi_{i}-\lambda_{2} \sin \varphi_{i}\right) \Delta_{i}$.

Suppose first that $\lambda_{1}, \lambda_{2} \neq 0$. The signs of the parameters $\lambda_{1}, \lambda_{2}$ determine those of $\operatorname{Re} a_{2}, \operatorname{Im} a_{2}$ belonging to the extremal function: the transformation $\left(\varphi_{1}, \varphi_{2}\right) \rightarrow$ 
$\left(-\varphi_{1},-\varphi_{2}\right)$ changes the sign of $\operatorname{Im} a_{2}$ and keeps $\operatorname{Re} a_{2}$ fixed, while the transformation $\left(\varphi_{1}, \varphi_{2}\right) \rightarrow\left(\varphi_{1}+\pi, \varphi_{2}+\pi\right)$ only changes the sign of $\operatorname{Re} a_{2}$. Thus always $-\lambda_{1} \operatorname{Re} a_{2}>0, \lambda_{2} \operatorname{Im} a_{2}>0$ in the maximum case.

The necessary conditions $\partial L / \partial \varphi_{i}=0, i=1,2$, give the equations

$$
\begin{aligned}
& \lambda_{1}=c_{1}+4 \operatorname{Re} a_{2}, \\
& \lambda_{2}=c_{2}+4 \operatorname{Im} a_{2},
\end{aligned}
$$

where $c_{1}$ and $c_{2}$ have the expressions

$$
c_{1}=4 \frac{\sin \varphi_{1}-\sin \varphi_{2}}{\sin \left(\varphi_{1}-\varphi_{2}\right)} \cos \varphi_{1} \cos \varphi_{2},
$$

$$
c_{2}=4 \frac{\cos \varphi_{1}-\cos \varphi_{2}}{\sin \left(\varphi_{1}-\varphi_{2}\right)} \sin \varphi_{1} \sin \varphi_{2},
$$

and $\operatorname{Re} a_{2}, \operatorname{Im} a_{2}$ are given by

$$
\begin{aligned}
& 2 \operatorname{Re} a_{2}=(k / 2+1) \cos \varphi_{1}-(k / 2-1) \cos \varphi_{2}, \\
& 2 \operatorname{Im} a_{2}=-(k / 2+1) \sin \varphi_{1}+(k / 2-1) \sin \varphi_{2} .
\end{aligned}
$$

Fix now the situation by setting $\lambda_{1}<0, \lambda_{2}>0$. Accordingly, we must have $\operatorname{Re} a_{2}>0$, Im $a_{2}>0$. If we denote $E=\left\{\left(\varphi_{1}, \varphi_{2}\right) \mid 3 \pi / 2<\varphi_{1}<2 \pi, \pi / 2<\varphi_{2}<\varphi_{1}-\pi\right\}$, then (10) gives the mapping from $E$ onto the points $\operatorname{Re} a_{2}>0, \operatorname{Im} a_{2}>0$ which belong to the part of $V_{3}$ produced by two-point measures; see [7]. For $\left(\varphi_{1}, \varphi_{2}\right) \in E$ we have $c_{1}<0, c_{2}>0$. The condition $\lambda_{2}=c_{2}+4 \operatorname{Im} a_{2}>0$ is thus satisfied. However, the sum $\lambda_{1}=c_{1}+4 \operatorname{Re} a_{2}$ is not negative for all $\left(\varphi_{1}, \varphi_{2}\right)$ in $E$. Especially, $\lambda_{1}$ is positive on the two lines $\varphi_{2}=\pi / 2,3 \pi / 2<\varphi_{1}<2 \pi$ and $\varphi_{1}=2 \pi, \pi / 2<\varphi_{2}<\pi$. These lines give the boundaries between the parts of $V_{3}$ produced by two-point and threepoint measures, so measures which are close to the three-point measures cannot maximize the functional (7).

It is not difficult to check that for all $\left(\varphi_{1}, \varphi_{2}\right) \in E$ the derivative of $\operatorname{Re} a_{2}$ and $c_{1}$ with respect to $\varphi_{1}$ are positive. Since $\lim _{\varphi_{1} \rightarrow\left(\varphi_{2}+\pi\right)+} c_{1}=-\infty$ for each $\varphi_{2} \epsilon$ $(\pi / 2, \pi)$, it follows that the equation $0=c_{1} / 4+\operatorname{Re} a_{2}$, i.e.,

$$
0=\frac{\sin \varphi_{1}-\sin \varphi_{2}}{\sin \left(\varphi_{1}-\varphi_{2}\right)} \cos \varphi_{1} \cos \varphi_{2}+\frac{1}{2}\left(\frac{k}{2}+1\right) \cos \varphi_{1}-\frac{1}{2}\left(\frac{k}{2}-1\right) \cos \varphi_{2},
$$

has a unique solution $\varphi_{1}^{0}=\varphi_{1}^{0}\left(\varphi_{2}, k\right), \varphi_{2}+\pi<\varphi_{1}^{0}<2 \pi$, for each fixed $\varphi_{2} \in(\pi / 2, \pi)$. For $\varphi_{1}^{0}<\varphi_{1}<2 \pi$ the sum $c_{1}+4 \operatorname{Re} a_{2}$ is positive, so a necessary condition for a pair $\left(\varphi_{1}, \varphi_{2}\right)$ to maximize the functional (7) with $\lambda_{1}<0, \lambda_{2}>0$ is given by $\varphi_{2}+\pi<\varphi_{1}<\varphi_{1}^{0}\left(\varphi_{2}, k\right)$.

Consider next the rotated functional

$$
L_{\tau}\left(\varphi_{1}, \varphi_{2}\right)=\operatorname{Re}\left\{\tau^{2} 6 a_{3}-\tau \lambda 2 a_{2}\right\}, \quad|\tau|=1 .
$$

By (6) a rotation $\varphi \rightarrow \varphi+t, \tau=e^{i t}$, on the unit circle transforms $a_{2} \rightarrow \tau^{-1} a_{2}, a_{3} \rightarrow \tau^{-2} a_{3}$, 
and so $L_{\tau}\left(\varphi_{1}+t, \varphi_{2}+t\right)=L\left(\varphi_{1}, \varphi_{2}\right)$. Hence we can extend outside $E$ the necessary condition for pairs $\left(\varphi_{1}, \varphi_{2}\right)$ to maximize a linear functional of $a_{2}, a_{3}$. For any $\varphi_{2}^{*} \in[0,2 \pi)$ we can select any arbitrary point $\varphi_{2} \in(\pi / 2, \pi)$ and consider the functional $L_{\tau}$ with $\arg \tau=\varphi_{2}^{*}-\varphi_{2}$. The necessary condition for a pair $\left(\varphi_{1}^{*}, \varphi_{2}^{*}\right), \varphi_{1}^{*}>\varphi_{2}^{*}+\pi$, to maximize the functional is given by the inequality

$$
\varphi_{2}^{*}+\pi<\varphi_{1}^{*}<\varphi_{2}^{*}+\pi+d\left(\varphi_{2}\right), d\left(\varphi_{2}\right)=\varphi_{1}^{0}\left(\varphi_{2}, k\right)-\varphi_{2}-\pi .
$$

The best scope for the condition is given by the maximum of $d\left(\varphi_{2}\right), \pi / 2<\varphi_{2}<\pi$. The right hand side of (11) is increasing with $k$, so

$$
c(k)=\max _{\varphi_{2}}\left(\varphi_{1}^{0}\left(\varphi_{2}, k\right)-\varphi_{2}-\pi\right)
$$

is a decreasing function of $k$. The condition for a pair $\left(\varphi_{1}, \varphi_{2}\right), \varphi_{2}+\pi<\varphi_{1}$, to give a maximum for a functional (12) with $\lambda_{1}<0, \lambda_{2}>0$, is given by $0<\varphi_{1}-\varphi_{2}-$ $\pi<c(k)$.

Consider then the functional (17) with the parameters in the quadrant $\lambda_{1}<0$, $\lambda_{2}<0$. For a maximizing function we now have $\operatorname{Re} a_{2}>0, \operatorname{Im} a_{2}<0$. These values are obtained from those of the preceding case by the transformation $\left(\varphi_{1}, \varphi_{2}\right) \rightarrow$ $\left(-\varphi_{1},-\varphi_{2}\right)$ which keeps $\operatorname{Re} a_{2}, c_{1}$ fixed and changes the signs of $\operatorname{Im} a_{2}, c_{2}$. For each $\varphi_{2} \in(-\pi / 2,-\pi)$ the equations (8) now have a solution if $0<\varphi_{2}+\pi-\varphi_{1}<$ $\varphi_{1}^{0}\left(\varphi_{2}, k\right)$. As above, we conclude the condition $0<\varphi_{2}-\varphi_{1}+\pi<c(k)$ for any $\left(\varphi_{1}, \varphi_{2}\right)$ to maximize the functional (12). Combining this with the previous inequality and denoting $x=e^{-i \varphi_{1}}, y=e^{-i \varphi_{2}}$, we have the necessary condition $0<|\arg (-x \bar{y})|<c(k)$ for the functions (3) to maximize the functional (12) with $\lambda_{1}, \lambda_{2} \neq 0$.

When $\lambda_{2}=0$ the functional (7) achieves its maximum by the functions (3) with $x=\pi, y=0$, or $x=0, y=\pi$, which simultaneously maximize $\operatorname{Re} a_{3}$ and $\left|\operatorname{Re} a_{2}\right|$.

For $\lambda_{1}=0, \lambda_{2}>0$ the equations (8) can be solved in terms of $\left(\varphi_{1}^{0}\left(\varphi_{2}, k\right), \varphi_{2}\right) \in E$ only when $\lambda_{2}$ is small enough. The upper limit for these $\lambda_{2}$ can be found by the Taylor expansions of the formulae in (8); cf. page 65. We omit here the computations. With larger values of $\lambda_{2}$ the extremal function is $f_{k}(z, x, y)$ with $\arg x=\pi / 2$, arg $y=3 \pi / 2$. The case $\lambda_{2}<0$ is symmetric with that of $\lambda_{2}>0$.

Remarks. The values of $c(k)$ are obtained numerically. For instance $c(2)=0.679674 \ldots, c(3)=0.528959 \ldots, c(4)=0.429386 \ldots$. The condition of the theorem could be compared to the result of Aharonov and Friedland [1], according to which the functions (3) are extreme points of $\overline{c o}\left(B_{k}\right), k \geqq 4$, at least if $|\arg (-x \bar{y})| \leqq 4 \pi / k$. The question arises whether all the functions (3) satisfying $|\arg (-x \bar{y})|<c(k)$, $2<k<4$, are extreme points and support points of $B_{k}$. This is true if the formulas (8) give a bijective map from $E_{0}=\left\{\left(\varphi_{1}, \varphi_{2}\right) \mid \pi / 2<\varphi_{2}<\pi, \varphi_{2}+\pi<\varphi_{1}<\varphi_{1}^{0}\left(\varphi_{2}, k\right)\right\}$ onto the set $T=\left\{\left(\lambda_{1}, \lambda_{2}\right) \mid \lambda_{1}<0, \lambda_{2}>0\right\}$. It can be seen that the mapping (8) is not injective in the set $E$. However, numerically one can verify that the injectivity indeed seems to hold in $E_{0}$. 
In the class $S$ the functional $\operatorname{Re}\left\{a_{3}-\lambda a_{2}\right\}$ was studied by Brown [6] and in the class $S(b)$ of bounded univalent functions by Tammi [13]. The functional generates a rather rich family of support points, since generally distinct values of $\lambda$ give distinct extremal functions [6]. Here we give a necessary condition for these support points.

The study is parallel to the above case of $B_{k}$ if we use the Lowner expressions

$$
\begin{gathered}
a_{2}=-2 \int_{0}^{1} \varkappa(u) d u, \\
a_{3}=a_{2}^{2}-2 \int_{0}^{1} u \varkappa(u)^{2} d u, \\
\varkappa(u)=e^{-i \theta(u)}, \quad 0 \leqq u \leqq 1 .
\end{gathered}
$$

The coefficient body $V_{3}$ of $S$ was obtained by Schaeffer and Spencer [12] by variational methods. In [7] the body was determined from the above formulae by maximization of the functional $\operatorname{Re}\left\{a_{3}-a_{2}^{2}-c a_{2}\right\}$ for varying $c, c \in C$. The body consists of two main cases, Part I and Part II. Part I, which consists of boundary functions of forked-slit type, is obtained with real parameters $c$. Part II consisting of one-slit boundary functions is obtained with parameter values $c=c_{1}+i c_{2}, c_{1}, c_{2} \neq 0$.

Since the support points of $S$ are known to be of one-slit type, it suffices to restrict to Part II. We recall the parametric presentation for these boundary functions.

In Part II the control function $\theta(u)$ for the Lowner equation of the boundary, i.e., the function maximizing $\operatorname{Re}\left\{a_{3}-a_{2}^{2}-c a_{2}\right\}$, satisfies the Euler equation

$$
u \sin 2 \theta(u)-c_{1} \sin \theta(u)+c_{2} \cos \theta(u)=0 .
$$

When, for instance, $c_{1}<0, c_{2}>0$, the equation gives a unique monotonic control function $\theta(u), \pi / 2<\theta(u)<\pi$.

Denote the terminal values of $\theta$ by $\alpha=\theta(1), \omega=\theta(0)$. By (13) the parameters $c_{1}, c_{2}$ assume the expressions

$$
\begin{gathered}
c_{1}=\frac{\sin 2 \alpha \cos \omega}{\sin (\alpha-\omega)}, \\
c_{2}=\frac{\sin 2 \alpha \sin \omega}{\sin (\alpha-\omega)} .
\end{gathered}
$$

In terms of $\alpha$ and $\omega$ the integrated expressions for $\operatorname{Re} a_{2}$ and $\operatorname{Im} a_{2}$ are

$$
\operatorname{Re} a_{2}=c_{1} \log \frac{\cos \alpha}{\cos \omega}+c_{2}(\cot \alpha-\cot \omega+\alpha-\omega),
$$

$$
\operatorname{Im} a_{2}=c_{2} \log \frac{\sin \alpha}{\sin \omega}+c_{1}(\tan \alpha-\tan \omega-\alpha+\omega) .
$$


The parameters $\alpha, \omega$ determine the boundary function in a unique way. If we denote $F=\{(\alpha, \omega) \mid \pi / 2<\alpha<\pi, \alpha<\omega<\pi\}$, the formulas (14) and (15) give the map from the set $F$ onto the points $\operatorname{Re} a_{2}>0, \operatorname{Im} a_{2}>0$ which belong to Part II.

Theorem 3. The functional $\operatorname{Re}\left\{\tau^{2} a_{3}-\tau \lambda a_{2}\right\}, \tau, \lambda \in C,|\tau|=1$, can reach its maximum in the set $S$ only by one-slit boundary functions of $V_{3}$ satisfying the additional condition

$$
|\theta(1)-\theta(0)| \leqq c,
$$

where $c=0.601394 \ldots$ and $\theta(0), \theta(1)$ denote the terminal values of the control function of the Lowner equation for the boundary function.

Proof. Start with the functional $L(\theta)=\operatorname{Re}\left\{a_{3}-\left(\lambda_{1}+i \lambda_{2}\right) a_{2}\right\}$, and suppose first that $\lambda_{1}, \lambda_{2} \neq 0$. The Lowner expressions give

$$
L(\theta)=4\left(\int_{0}^{1} \cos \theta d u\right)^{2}-4\left(\int_{0}^{1} \sin \theta d u\right)^{2}-2 \int_{0}^{1}\left(u \cos 2 \theta-\lambda_{1} \cos \theta-\lambda_{2} \sin \theta\right) d u .
$$

The necessary extremum condition, obtained by a variation of $\theta$ (or by formal differentiation with respect to $\theta$ ) reads

$$
u \sin 2 \theta-\left(\lambda_{1} / 2-\operatorname{Re} a_{2}\right) \sin \theta+\left(\lambda_{2} / 2-\operatorname{Im} a_{2}\right) \cos \theta=0 .
$$

Comparison with (13) gives the necessary conditions

$$
\begin{aligned}
& \lambda_{1} / 2=c_{1}+\operatorname{Re} a_{2}, \\
& \lambda_{2} / 2=c_{2}+\operatorname{Im} a_{2},
\end{aligned}
$$

where $c_{1}, c_{2}$ and $\operatorname{Re} a_{2}, \operatorname{Im} a_{2}$ are given in terms of $\alpha, \omega$ as in (14) and (15).

Again the signs of $\lambda_{1}, \lambda_{2}$ fix those of $\operatorname{Re} a_{2}, \operatorname{Im} a_{2}$ belonging to the function maximizing $L$. For $\lambda \in T=\left\{\left(\lambda_{1}, \lambda_{2}\right) \mid \lambda_{1}<0, \lambda_{2}>0\right\}$ we must have $\operatorname{Re} a_{2}>0, \operatorname{Im} a_{2}>0$. These values correspond to $(\alpha, \omega) \in F$, for which $c_{1}<0, c_{2}>0$. The condition $\lambda_{2} / 2=c_{2}+\operatorname{Im} a_{2}>0$ is thus satisfied, while the inequality $\lambda_{1} / 2=c_{1}+\operatorname{Re} a_{2}<0$ is not satisfied by all $(\alpha, \omega) \in F$. Especially, the sum is positive on the two lines given by $\alpha=\pi / 2, \pi / 2<\omega<\pi$ and $\pi / 2<\alpha<\pi, \omega=\pi$. These lines give the boundary between Part I and Part II in the quadrant $\operatorname{Re} a_{2}>0, \operatorname{Im} a_{2}>0$, whence the functions of Part II which are too close to Part I cannot maximize any functional (16).

The expressions of $c_{1}$ and $\operatorname{Re} a_{2}$ are increasing with $\omega$ for each $\pi / 2<\alpha<\omega$. Because $\lim _{\omega \rightarrow \alpha+} c_{1}=-\infty$, the equation $0=c_{1}+\operatorname{Re} a_{2}$ has a unique solution $\omega^{0}=\omega^{0}(\alpha), \alpha<\omega^{0}<\pi$, for each fixed $\alpha$. When $\omega^{0}<\omega<\pi / 2$, the expression $c_{1}+\operatorname{Re} a_{2}$ is positive, so a necessary condition for $(\alpha, \omega) \in F$ to maximize (16) with $\lambda_{1}<0, \lambda_{2}>0$ is given by $\alpha<\omega<\omega^{0}(\alpha)$.

Consider then the rotated functional $L_{\tau}(\theta)=\operatorname{Re}\left\{\tau^{2} a_{3}-\tau \lambda a_{2}\right\}, \tau=e^{i t}$. Replacing the function $\theta$ by $\tilde{\theta}(u)=\theta(u)+t$ in Lowner's formulae we get $\tilde{a}_{2}=\tau^{-1} a_{2}, \tilde{a}_{3}=\tau^{-2} a_{3}$. So $L_{\tau}(\tilde{\theta})=L(\theta)$, and the maxima of $L$ and $L_{\tau}$ coincide for $\theta$ varying over the 
set of admissible functions. Similarly to the case of $B_{k}$ we deduce the necessary condition for $\tilde{\theta}$ to maximize some functional $L_{\tau}$ : the functions $\tilde{\theta}(u)-t$ must satisfy (13) for some constant $t$, and the terminal values of $\tilde{\theta}$ have to satisfy $0<|\tilde{\theta}(1)-\tilde{\theta}(0)|<c$, where $c=\max _{\alpha \in(\pi / 2, \pi)} \omega^{0}(\alpha)-\alpha$. The numeric value of $c$ is obtained by computer.

Finally, consider the cases where one of the parameters $\lambda_{1}, \lambda_{2}$ vanishes. If $\lambda_{2}=0$, the maximum $3+2\left|\lambda_{1}\right|$ for $L$ is reached by the Koebe function. If $\lambda_{1}=0$, $\lambda_{2}>0$, the equations (17) have a solution $\left(\alpha, \omega^{0}(\alpha)\right) \in F$ only when $\lambda_{2}<4 e /(e-1)$; cf. [13] where Tammi determined the constant for the class $S(b)$. For $\lambda_{2}>4 e /(e-1)$ the extremal function is the (rotated) Koebe function. The case of a negative $\lambda_{2}$ is symmetric with that of a positive $\lambda_{2}$. The Koebe function is obtained from the Lowner equation by a constant control function, i.e., by a $\theta$ satisfying $\theta(1)=\theta(0)$.

Remarks. The converse to the theorem holds if (17) gives a bijective mapping between $T$ and the set $F_{0}=\left\{(\alpha, \omega) \mid \pi / 2<\alpha<\pi, \alpha<\omega<\omega^{0}(\alpha)\right\}$. The mapping is not injective in the set $F$. Numerical verification indicates that the restriction to the set $F_{0}$ would be injective, but due to the implicit character of the formulae and the set $F_{0}$ we do not have an exact proof for this.

Schaeffer and Spencer showed that the support points of the body $V_{n}, n>2$, form a closed connected subset of the boundary points of $V_{n}$ corresponding to one-slit functions ([12], Chapter X). The condition of Theorem 3 could be regarded as a quantitative example of this general result.

\section{References}

[1] Aharonov, D., and S. Friedland: On functions of bounded boundary rotation. - Ann. Acad. Sci. Fenn. Ser. A I Math. 585, 1974, 1-17.

[2] Brannan, D. A., J. G. Clunie and W. E. Kirwan: On the coefficient problem for functions of bounded boundary rotation. - Ann. Acad. Sci. Fenn. Ser. A I Math. 523, 1973, 1-18.

[3] Brickman, L.: Extreme points of the set univalent functions. - Bull. Amer. Math. Soc. 76, $1970,372-374$.

[4] Brickman, L., T. H. MacGregor, and D. R. Wilken: Convex hulls of some classical families of univalent functions. - Trans. Amer. Math. Soc. 156, 1971, 91-107.

[5] Brickman, L. and D. Wilken: Subordination and insuperable elements. - Michigan Math. J. 23, 1976, 225-233.

[6] Brown, J. E.: Univalent functions maximizing $\operatorname{Re}\left\{a_{3}+\lambda a_{2}\right\}$. - Illinois J. Math. 25, 3, 1981. $446-454$.

[7] HaArio, H.: On coefficient bodies of univalent functions. - Ann. Acad. Sci. Fenn. Ser A I Math. Dissertationes 22, 1978, 1-49.

[8] HaARIO, H.: On the range of real coefficients of functions with bounded bounded boundary rotation. - Ann. Acad. Sci. Fenn. Ser. A I Math. 7, 1982,

[9] Hallenbeck, D. J.: Convex hulls and extreme points of some families of univalent functions. Trans. Amer. Math. Soc. 192, 1974, 285-292.

[10] Hengartner, W., A. Pfluger and G. Schober: On support points in the class of functions with bounded boundary rotation. - Ann. Acad. Sci. Fenn. Ser. A I Math. 6, 1981, 213-224. 
[11] Pfluger, A.: Lineare Extremalprobleme bei schlichten Funktionen. - Ann. Acad. Sci. Fenn. Ser. A I Math. 489, 1971, 1-32.

[12] Schaeffer, A. C. and D. C. Spencer: Coefficient regions for schlicht functions. - American Mathematical Society Colloquium Publications 35, American Mathematical Society, New York, N. Y., 1950.

[13] TAMMI, O.: Extremum problems for bounded univalent functions II. - Lecture Notes in Mathematics 913, Springer Verlag, Berlin-Heidelberg-New York, 1982.

University of Helsinki

Department of Mathematics

SF-00100 Helsinki 10

Finland

Received 13 September 1982 